\title{
Effectiveness of Micropulse Mp3 Cyclodiode Laser in Controlling Intraocular Pressure without Acetazolamide
}

Imran Ghayoor, Sahira Wasim, Munira Shakir, Shakir Zafar

DOI 10.36351/pjo.v35i4.935

Pak J Ophthalmol 2019, Vol. 35, No. 4

See end of article for

authors affiliations

Correspondence to:

Dr. Sahira Wasim

Department of Ophthalmology,

Liaquat National Hospital Karachi

Email: Sahirawasim@gmail.com
Purpose: To determine the effectiveness of micropulse $\mathrm{mp} 3$ cyclodiode laser in controlling intraocular pressure without acetazolamide.

Study Design: Descriptive case series.

Place and Duration of Study: Department Ophthalmology, Liaquat National Hospital, Karachi for 6 months duration from 15-03-19 to 15-09-19.

Material and Methods: All patients of either gender with age 20 to 50 years with Primary open angle glaucoma, Neovascular, Refractory, Uveitic, Trauma induced glaucoma and Post vitrectomy glaucoma were included. Patients with Primary angle closure and Normal tension glaucoma were excluded from the study. Descriptive statistics were calculated. Frequencies and percentages were computed for qualitative variables. Quantitative variables were presented as mean \pm standard deviation. The mean baseline IOP was compared with mean IOP of 3 months using student t-test. Effect modifiers were controlled through stratification. Fisher exact test was used to see the association of effectiveness with stratified groups. P-value $\leq 0.05$ was considered as significant.

Results: Out of 98 patients included in the study, $63.3 \%$ were males and $36.7 \%$ were females. Mean age of the patients was $48.46 \pm 13.39$ years. The effectiveness of Micropulse Mp3 Cyclodiode Laser was observed in $85.7 \%$ cases. Significant mean difference was found between pre-op IOP with IOP after 3 months for right eye and left eye. Insignificant association of effectiveness was 
found with gender, age, type and procedure.

Conclusion: Micro pulse MP3 provides promising results with high level of effectiveness and with great potential advantages to be considered as a safe alternative procedure.

Key Words: Micropulse Mp3 Cyclodiode Laser, Intraocular Pressure,

Acetazolamide.

G laucoma is leading towards the second most common cause of worldwide irreversible blindness $^{1}$ and approximately sixty million people are suffering from glaucoma globally ${ }^{1,2}$. Glaucoma therapies are designed to either increase the outflow or decrease the production of aqueous humor in order to reduce intraocular pressure (IOP) and preserve visual function ${ }^{3}$. Studies have shown that reducing intraocular pressure helps to preserve visual function in most cases ${ }^{3}$. Surgical intervention is needed when medication fails to control intraocular pressure (IOP), which is required to preserve optic nerve function ${ }^{4}$. Current glaucoma therapies include topical medications, laser therapies, microinvasive glaucoma surgery, and incisional glaucoma surgery. Most therapies are designed to reduce the production of aqueous humor, increase uveoscleral outflow or both $^{5}$.

Trabeculectomy with or without anti-metabolites, and glaucoma drainage devices are considered to be the initial IOP lowering surgical procedures followed by ${ }^{6}$ Cycloablation, in which destruction of ciliary body epithelium and stroma is done, thus reducing aqueous production7. Cyclo G6 system with MP3 probe, deliver microsecond thermal energy that is confined to target tissue, preventing destruction of surrounding tissue by on and off cycles mode, allowing energy to build up in the targeted pigmented tissues, reaching to coagulative threshold?.

In Ten et al study the mean preoperative IOP was $39.3 \pm 12.6 \mathrm{~mm} \mathrm{Hg}$ that decreased to $31.1 \pm 13.4 \mathrm{~mm}$ $\mathrm{Hg}, 28.0 \pm 12.0 \mathrm{~mm} \mathrm{Hg}, 27.4 \pm 12.7 \mathrm{mmHg}, 27.1 \pm 13.6$ $\mathrm{mm} \mathrm{Hg}, 25.8 \pm 14.5 \mathrm{~mm} \mathrm{Hg}, 26.6 \pm 14.7 \mathrm{~mm} \mathrm{Hg}$ and $26.2 \pm 14.3 \mathrm{~mm} \mathrm{Hg}$ at 1 st day, $1^{\text {st }}$ week, 1, 3, 6, 12 and 18 months respectively. After a mean of 1.3 treatment sessions, success achieved was $72.7 \%{ }^{8}$. Numerous studies have demonstrated the efficacy and high safety profile of micro pulse trans-scleral cyclophotocoagulation MP-TSCPC in refractory glaucomas ${ }^{9-14}$. Reduction of mean IOP was seen in $60.3 \%$ at 1 week and $33.4 \%$ at 1 month. The procedure was safe in all cases and effectiveness was found in $71 \%$ of the patients ${ }^{15}$.

There are few international studies in literature describing the clinical outcomes of micropulsed mp3 cyclodiode laser, in which the work is mostly done in patients with advanced glaucoma with no local studies. The aim of this study is to consider MP3 cyclodiode laser for other glaucoma patients, to control intraocular pressure with the reduction of number of treatments especially excluding the oral acetazolamide to minimize collateral damage, unwanted side effects and to overcome the unavailability of this drug in Pakistan.

\section{MATERIAL AND METHODS}

This descriptive case series was conducted from 15.03.19 to 15.09.19 at Liaquat National Hospital, Karachi in the department of ophthalmology after the approval of ethical committee. WHO sample size calculator was used to calculate sample size. All patients of either gender with age 20 to 50 years having Primary open angle glaucoma, Neovascular, Refractory, Uveitic, Trauma induced glaucoma, and Post vitrectomy induced glaucoma were included in the study. Patients with Primary angle closure and Normal tension glaucoma were excluded from the study.

Clinical history was recorded. Informed written consent was taken before enrolment. Data was collected using a proforma, which included age, gender, duration of glaucoma, number of antiglaucoma medications, visual acuity using Snellen chart and intraocular pressure with the Goldmann Applanation tonometer. Type of glaucoma was labeled after slit lamp examination. The Micro pulse trans-scleral cyclophotocoagulation (MPTSCPC) diode laser procedure was performed after injecting retro bulbar anesthesia of 3-5ml of lidocaine. Cyclo G6 laser system (IRIDEX laser system) which uses a laser diode 
of $810 \mathrm{~nm}$ infrared wavelength with MP3 probe was used. Treatment was done using total duration of 1.6 millisecond (ms) including 0.5 millisecond on time, 1.1 ms off time, $31.33 \%$ duty cycle and power of $2000 \mathrm{~mW}$. Globe manipulation by cotton swabs and placement of speculum was censured by adequate exposure to the targeted area. The laser probe was positioned perpendicular to the surface of the globe with fiberoptic tip $3 \mathrm{~mm}$ away from the limbus. Laser application was done to the upper and lower hemisphere in "painting" direction, avoiding the 3 and 9 o'clock positions to avoid risk of damage to the neurovascular bundles. The laser was delivered for 80 seconds for superior and inferior hemisphere for a total of 160 seconds of treatment. Patients received post-operative dose of dexamethasone ointment and were patched for 1 hour. All patients were started on topical Moxifloxicin, Fluoromethalone and neomycin one hourly and after 1 week tapered to 4 times a day. The following baseline parameters were collected for each visit at 1 week, 1 month and 3 months. Intraocular pressure, number of anti-glaucoma medications used including oral acetazolamide and any complications were recorded. Topical antiglaucoma medications were tapered or adjusted at the doctor's discretion. Effectiveness of the procedure of the treated eyes was defined as reduction of IOP by $30 \%$ from baseline IOP after 1 month follow up or withdrawal of oral acetazolamide.

SPSS version 22 was used for data compilation and analysis. Frequencies and percentages were computed for categorical variables. Quantitative variables were presented as mean \pm standard deviation. The mean baseline IOP was compared with mean IOP at 3 months using student $t$ test. Effect modifiers were controlled through stratification. Poststratification chi square and fisher exact test was used to see the association of effectiveness with stratified groups. Repeated measures of ANOVA were applied to compare means. $P$ value $\leq 0.05$ was considered level of significance.

\section{RESULTS}

Ninety-eight cases were included in study. Out of whom $62(63.3 \%)$ were males and $36(36.7 \%)$ were females. The descriptive statistics including mean age of the patients, type of glaucoma, side of treatment, quadrants treated are given in Table 1. Effectiveness of treatment was seen in $85.7 \%$ cases. We found insignificant association of effectiveness with gender $(p=0.199)$, age groups $(p=0.096)$, type $(p=0.656)$ and procedure $(\mathrm{p}=0.231)$ as shown in Table-2.

Mean pre-op IOP, after 1 week, 1 month and 3 months for unilateral and bilateral cases is shown in Table-3. Acetazolamide was not given to $35(35.7 \%)$ cases while stopped for $53(54.1 \%)$ cases and 10(10.2\%) cases continued with acetazolamide.

Mean post-operative IOP at each time point was significantly lower than pre op IOP for unilateral (right and left eye) and bilateral (right and left eye) cases as shown in Figure 1 (a), Figure 1 (b), Figure 2 (a) and Figure $2(b)$. Significant mean difference was found for pre-op IOP with IOP after 3 months for unilateral right eye $(\mathrm{p}=0.000)$, unilateral left eye $(\mathrm{p}=$ $0.00)$, Bilateral right eye $(p=0.000)$ and Bilateral left eye $(p=0.000)$ as presented in Table- 4 .

Our complications which were generally tolerated well were conjunctival hemorrhage because of the tip of the probe, which resolved later. The most significant but rare side effect seen was severe surface epithelial erosion all over cornea and it took 3-6 weeks to come back to normal with Autologus serum. In one patient, permanent central scar was formed because of infection. Four to five patients did not respond to treatment even after repeating the procedure after 3 months. We could not explain this phenomenon. 
Table 1: Descriptive statistics of study population.

\begin{tabular}{llc}
\hline & & $\mathbf{n}(\%)$ \\
\hline Age(years) & & \\
Gender & Male & $48.46 \pm 13.39$ \\
Number of Drops & Female & $62(63.3)$ \\
& 0 to 0 & $36(36.7)$ \\
& 1 to 1 & $1(1)$ \\
& 2 to 0 & $16(16.3)$ \\
& 2 to 1 & $9(9.2)$ \\
& 2 to 2 & $11(11.2)$ \\
& 3 to 0 & $22(22.4)$ \\
AZM & 3 to 1 & $6(6.1)$ \\
& 3 to 2 & $8(8.2)$ \\
Type & 3 to 3 & $17(17.3)$ \\
& 4 to 2 & $7(7.1)$ \\
& Stop & $1(1)$ \\
& Continue & $53(54.1)$ \\
& Not Given & $10(10.2)$ \\
& Chronic & $35(35.7)$ \\
& Neovascular & $18(18.4)$ \\
& Refractory & $4(4.1)$ \\
Eye & Trauma & $14(28.6)$ \\
& Uveitic & $6(6.1)$ \\
& Vitrectomy & $28(28.6)$ \\
& Right & $36(36.7)$ \\
& Left & $36(36.7)$ \\
& Both & $26(26.5)$ \\
& &
\end{tabular}

$\begin{array}{lll}\text { Procedure } & 180 & 34(34.7) \\ \text { Effectiveness } & 360 & 64(65.3) \\ & \text { Yes } & 84(85.7) \\ & \text { No } & 14(14.3)\end{array}$

Mean \pm SD

Table 2: Association of effectiveness with population characteristics.

\begin{tabular}{llccc}
\hline & & \multicolumn{2}{c}{ Effectiveness } & P- \\
& & Yes & No & value \\
\hline Gender & Male & $51(60.7)$ & $11(78.6)$ & \multirow{2}{*}{0.199} \\
& Female & $33(39.3)$ & $3(21.4)$ & \\
Age Group & $\leq 50$ years & $34(40.5)$ & $9(64.3)$ & 0.096 \\
Type $\_$ & $>50$ years & $50(59.5)$ & $5(35.7)$ & \\
& Chronic & $16(19)$ & $2(14.3)$ & \\
& Neovascular & $4(4.8)$ & $0(0)$ & \\
& Refractory & $25(29.8)$ & $3(21.4)$ & 0.656 \\
& Trauma & $11(13.1)$ & $3(21.4)$ & \\
& Uveitic & $4(4.8)$ & $2(14.3)$ & \\
& Vitrectomy & $24(28.6)$ & $4(28.6)$ & \\
Procedure $\uparrow$ & 180 degree & $27(32.1)$ & $7(50)$ & 0.231 \\
\hline & 360 degree & $57(67.9)$ & $7(50)$ & \\
\hline
\end{tabular}

Chi Square test was applied.

$\uparrow$ Fisher exact test was applied.

$\mathrm{P} \leq 0.05$, considered as significant.

Table 3: IOP according to right and left eye.

\begin{tabular}{lcccc}
\hline & \multicolumn{2}{c}{ Unilateral $(\mathbf{n}=\mathbf{7 4})$} & \multicolumn{2}{c}{ Bilateral $(\mathbf{n}=\mathbf{4 9 )}$} \\
Right Eye $(\mathbf{n}=\mathbf{4 0})$ & Left Eye $(\mathbf{n}=\mathbf{3 4})$ & Right Eye $(\mathbf{n}=\mathbf{2 5})$ & Left Eye $(\mathbf{n}=\mathbf{2 4})$ \\
\hline Pre op IOP & $31.22 \pm 13.28$ & $34.05 \pm 13.39$ & $26.68 \pm 11.99$ & $25.75 \pm 7.99$ \\
1st week IOP & $16.13 \pm 5.87$ & $22.23 \pm 12.65$ & $14.72 \pm 5.79$ & $14.83 \pm 4.47$ \\
1st Month IOP & $14.30 \pm 5.21$ & $18.85 \pm 12.61$ & $13.52 \pm 4.20$ & $13.41 \pm 3.07$ \\
3rd Month IOP & $12.73 \pm 4.73$ & $16.79 \pm 12.54$ & $11.60 \pm 3.68$ & $11.12 \pm 2.00$ \\
\hline
\end{tabular}

Table 4: Mean difference of IOP at pre-op and after 3 months.

\begin{tabular}{|c|c|c|c|c|c|c|c|}
\hline & \multicolumn{3}{|c|}{ Unilateral } & \multicolumn{3}{|c|}{ Bilateral } \\
\hline & & Mean & SD & $P$-value & Mean & SD & P-value \\
\hline \multirow{2}{*}{ Left Eye } & Pre op IOP & 34.05 & 13.39 & \multirow{2}{*}{$<0.001$} & 25.75 & 7.99 & \multirow{2}{*}{$<0.001$} \\
\hline & IOP after 3 months & 16.79 & 12.54 & & 11.12 & 2.00 & \\
\hline \multirow{2}{*}{ Right Eye } & Pre op IOP & 31.23 & 13.28 & \multirow{2}{*}{$<0.001$} & 26.68 & 11.99 & \multirow{2}{*}{$<0.001$} \\
\hline & IOP after 3 months & 12.73 & 4.73 & & 11.60 & 3.68 & \\
\hline
\end{tabular}

Paired t-test was applied.

$\mathrm{P} \leq 0.05$, considered as significant. 


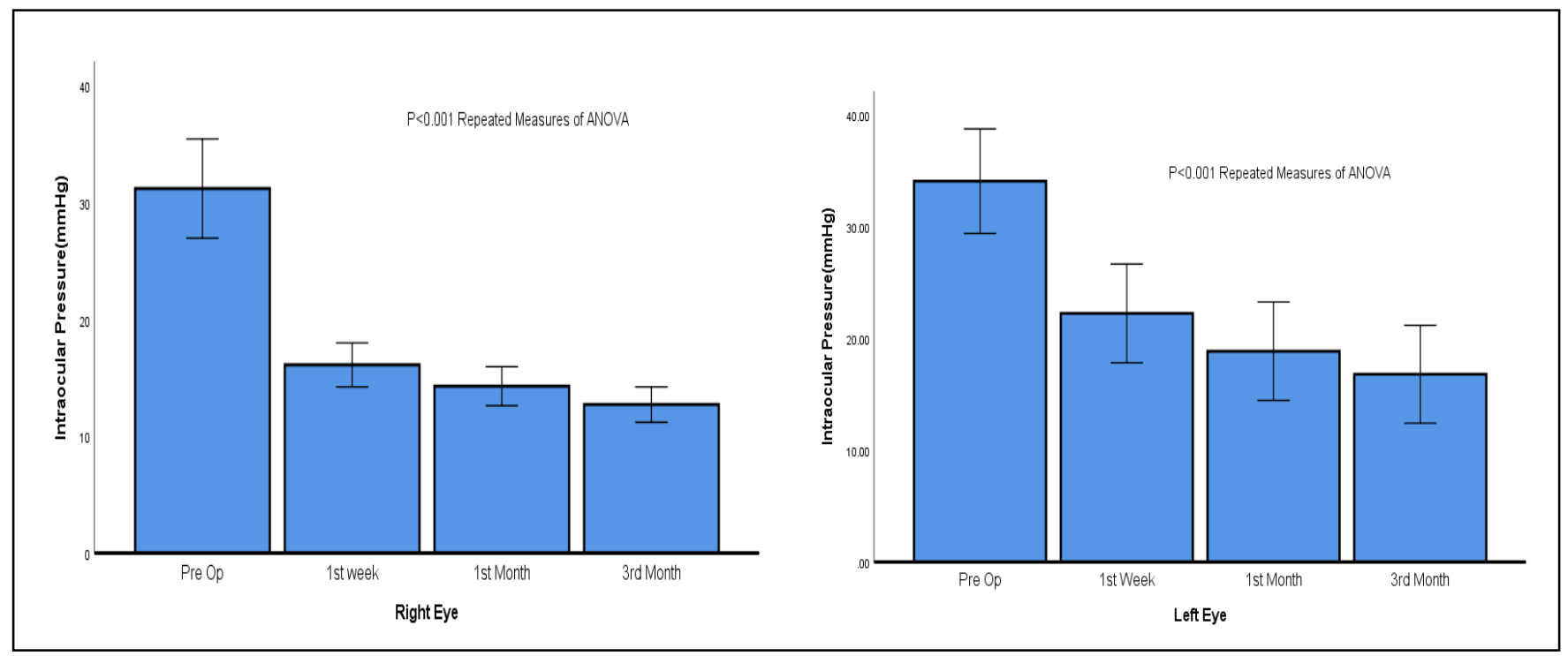

Fig. 1:
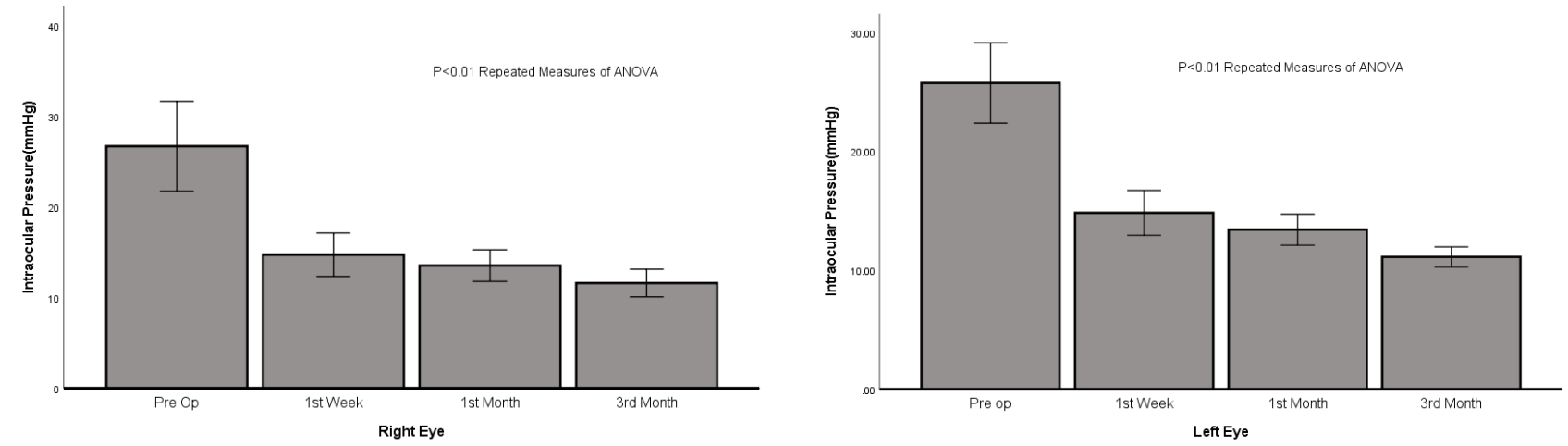

Fig.-2(a) and 2(b): Mean post-operative IOP at each time point is significantly lower than pre op IOP for right and left eye in bilateral cases $(\mathrm{p}<0.001)$. ANOVA indicates analysis of variance.

\section{DISCUSSION}

Cyclo photocoagulation (CPC) with MicroPulse 3 device represents a new tissue-sparing technology used for simple as well as for complex glaucoma ${ }^{15}$. Standard coagulation involves ciliary body epithelium and stroma destruction by targeting it, resulting in decreased aqueous secretion and eventually IOP control. As compared to conventional CPC which delivers continuous, high intensity energy, MP3 delivers repetitive short pulse laser energy series followed by rest period $8,15,16-18$. Complications related to cyclodestruction procedure includes vision loss, pupillary distortion, corneal edema, cystoid macula, hypotony, and edema ${ }^{19,20}$.
Micro pulse MP3 cyclophotocoagulation showed effectiveness for $85.7 \%$ of the cases in our study which is nearly same as reported by Kareen Zaroor $(81.7 \%)^{21}$. Yelenskiy A reported 71\% effectivness. ${ }^{19}$ Success rate varies from $40 \%$ to $80 \%$ in different studies $22-24$.

The advent of micro-pulsed trans-scleral diode laser has revolutionized diode laser as well as other laser types, even $\mathrm{CO}_{2}$ laser. Concept of micro-pulsing allows maximum effectiveness by generating significant amounts of energies to reach target tissues and allowing time for heat to diffuse instead of building up, to reduce the risk of unwanted side effects and to make this laser safe and predictable 
enough to use in seeing eyes ${ }^{1}$. The procedure is wellknown for its ease, non-invasiveness and well toleration. Bleeding and postoperative infection risks are eliminated by trans-scleral application. At every level of the glaucoma spectrum, MP3 is shown as safe and effective procedure for affected eyes ${ }^{1}$.

Excellent safety profile is documented with this treatment. One of the recent studies also reported very good results of the procedure with no complications (i.e. phthisis bulbi, hypotony and macular edema). ${ }^{6}$ In our study, we found significant mean difference for pre-op IOP with IOP after 3 months for right eye $(\mathrm{p}=$ $0.000)$ and left eye $(p=0.00)$. Emanuel et al. showed higher reduction of $\mathrm{IOP}^{24}$, this has been attributed both to the possibility of increased uveoscleral outflow, as well as decreased aqueous production ${ }^{22}$.

Emanuel et al also reported reduction in the need of topical eye drops ${ }^{21}$. Other studies also reported lesser need of number of eye drops ${ }^{10}$. However, it is noteworthy that in $54 \%$ of the patients in our study we were able to withdraw acetazolamide tablets, a treatment that was not used in other studies $1,16,24$. This could explain the reason why the number of hypotensive drops did not decrease as drastically as reported in other studies ${ }^{24}$.

The limitation of our study is the short-term follow up but we are continuing our study for long term follow-up. Previously, cyclodestructive procedures such as cyclocryotherapy and CPC were reserved for poorly controlled glaucoma, limited visual prognosis and mainly retained for end stage glaucoma, because of associated complication with cyclodestructive procedures that include vision loss, corneal edema, pupillary distortion, cystoid macula edema, and hypotony ${ }^{19}$. We did not notice any significant complications following MP-TSCPC in our study. Tan et al found MP-TSCPC comparable to conventional TSCPC with potentially lower rate of complication $^{23}$.

\section{CONCLUSION}

This new method of micropulse delivery may be of help in patients who cannot take medications or want to delay incisional surgery. Micro pulse MP3 can thus be a viable option in patients with prior failed filtering surgery, given the fact that repetition of incisional glaucoma procedures can be technically demanding and fraught with complications, not to mention the lower success rates of glaucoma reoperations. Micro pulse MP3 provides promising results with high level of effectiveness and with great potential advantages to be considered as a safe alternative procedure.

\section{CONFLICT OF INTEREST}

None

\section{REFERENCES}

1. Toyos MM, Toyos R. Clinical outcomes of micropulse transcleral cyclophotocoagulation in moderate to severe glaucoma. J Clin Exp Ophthalmol. 2016; 7 (620): 2.

2. Quigley HA, Broman AT. The number of people with glaucoma worldwide in 2010 and 2020. Br J Ophthalmol. 2006; 3: 262-7.

3. Weinreb RN, Khaw PT. Primary open-angle glaucoma. The Lancet. 2004; 363 (9422):1 711-20.

4. Lai JS, Tham CC, Lam DS. Surgical management of chronic closed angle glaucoma. Asian Pac J Ophthalmol. 2003; 15: 5-10.

5. Nguyen QH. Primary surgical management refractory glaucoma: tubes as initial surgery. Curr Opin Ophthalmol. 2009; 20: 122-5.

6. Noecker RJ, Kelly T, Patterson E, Herrygers LA. Diode laser contact trans-sclera cyclophotocoagulation: getting the most from the G - probe. Ophthalmic Surg Lasers Imaging, 2004; 35: 124-30.

7. Abdelrahman AM. Refractory Glaucomas. Types and Management. J Ophthalmol related Sci. 2017; 1 (1): 1-14.

8. Aquino MC, Barton K, Tan AM, Snq C, Li X, Loon SC, et al. Micropulse versus continuous wave transscleral diode cyclophotocoagulation in refractory glaucoma: a randomized exploratory study. Clin Exp Ophthalmol. 2015; 43 (1): 40-46.

9. Radcliffe N, Vold S, Kammer J. Micropulse transscleral cyclophotocoagulation (mTSCPC) for the treatment of glaucoma using the Micro Pulse P3 device. Am Glaucoma Soc annual meeting. 2015.

10. Kuchar S, Moster M, Waisbourd M. Treatment outcomes of Micro Pulse trans-scleral cyclophotocoagulation advanced glaucoma. Am Glaucoma Soc annual meeting, 2015.

11. Resende A, Waisbourd M, Amarasekera D. A prospective pilot study evaluating the novel Micropulse transscleral cyclophotocoagulation: short-term results. Am Glaucoma Soc annual meeting, 2016.

12. Lin S, Babic K, Masis M. Micropulse transscleral diode laser cyclophotocoagulation: short term results and anatomical effects. Am Glaucoma Soc annual meeting, 2016.

13. Maslin JS, Chen P, Sinard J, Noecker R. Comparison of acute histopathological changes in human cadaver eyes after MicroPulse and continuous wave transscleral cyclophotocoagulation. Am Glaucoma Soc annual meeting, 2016. 
14. Maslin J, Noecker R. Micropulse trans-scleral cyclophotocoagulation for the treatment of glaucoma. Assoc Res Vision Ophthalmol. 2016.

15. Gavris MM, Olteanu I, Kantor E, Mateescu R, Belicioiu R. IRIDEX MicroPulse P3: innovative cyclophotocoagulation. Romanian J Ophthalmol. 2017; 61 (2): 107.

16. Tan AM, Chockalingam M, Aquino MC, Lim Zl, See JL, Chew PT. Micropulse trans-scleral diode laser cyclophotocoagulation in the treatment of refractory glaucoma. Clin Exp Ophthalmol. 2010; 38 (3): 266-72.

17. Sivaprasad S, Sandhu R, Tandon A, Sayed Ahmed K, McHugh DA. Subthreshold micropulse diode laser

\section{Author's Affiliation}

Dr. Imran Ghayoor

Professor and Consultant

Department of Ophthalmology, Liaquat National Hospital, Karachi

Dr. Sahira Wasim

Resident

Department of Ophthalmology

Liaquat National Hospital, Karachi

Dr. Munira Shakir

Associate Professor

Department of Ophthalmology, Liaquat National Hospital, Karachi

Dr. Shakir Zafar

Associated Professor

Department of Ophthalmology, UMDC photocoagulation for clinically significant diabetic macular oedema: a three- year follow up. Clin Exp Ophthalmol. 2007; 35 (7): 640-4.

18. Parodi MB, Di Stefano G, Ravalico G. Grid laser treatment for exudative retinal detachment secondary to ischemic branch retinal vein occlusion. Retina. 2008; 28 (1): 97-102.

19. Yelenskiy A, Gillette TB, Arosemena A, Stern AG, Garris WJ, Young CT, et al. Patient Outcomes Following Micropulse Transscleral Cyclophotocoagulation: Intermediate-term Results. J glaucoma. 2018; 27 (10): 920-5.

20. Vernon SA, Koppens JM, Menon GJ, Negi AK. Diode laser cycloablation in adult glaucoma: long-term results of a standard protocol and review of current literature. Clin Exp Ophthalmol. 2006; 34 (5): 411-20.

21. Zaarour K, Abdelmassih Y, Arej N, Cherfan G, Tomey KF, Khoueir Z. Outcomes of Micropulse Transscleral Cyclophotocoagulation in Uncontrolled Glaucoma Patients. J Glaucoma. 2019; 28 (3): 270-5.

22. Kuchar S, Moster MR, Reamer CB, Waisbourd M. Treatment outcomes of micropulse transscleral cyclophotocoagulation in advanced glaucoma. Lasers Med Sci. 2016; 31 (2): 393-6.

23. Aquino MC, Barton K, Tan AM, Sng C, Li X, Loon SC, et al. Micropulse versus continuous wave transscleral diode cyclophotocoagulation in refractory glaucoma: a randomized exploratory study. Clin Exp Ophthalmol. 2015; 43 (1): 40-6.

24. Emanuel ME, Grover DS, Fellman RL, Godfrey DG, Smith O, Butler MR, et al. Micropulse cyclophotocoagulation: initial results in refractory glaucoma. J Glaucoma. 2017; 26 (8): 726-9.

\section{Author's Contribution}

Dr. Imran Ghayoor

Supervisor, surgeon \& advising consultant, Manuscript writing and final review

Dr. Sahira Wasim

Researcher, data analysis and final review

Dr. Munira Shakir

Surgeon, data analysis and final review

Dr. Shakir Zafar

Statistics, data analysis and final review 